\title{
Fluvoxamine in the treatment of trichotillomania, obsessive compulsive disorder and major depression -
}

\author{
a case report
}

\section{R Verster, MB C hB, DMH}

University of the Free State, Bloemfontein

M Matjane, MB ChB, MMed (Psy)

3 Military Hospital, Bloemfontein

Trichotillomania (TTM ), an impulse control disorder, and obsessive compulsive disorder ( $O C D$ ) have been studied as variants of the same spectrum of disorders. ${ }^{1,2}$ Arguments for and against this have been published. ${ }^{3} 0$ ther common problems that $O C D$ and TTM share are high co-morbidity rates $w$ ith other disorders ${ }^{46}$ and the treatment resistance experienced when TTM occurs as a single entity or in conjunction with OCD.4,7

Multiple treatment regimens for TTM and augmentation strategies have been postulated and researched, leading to no conclusive evidence of a fixed treatment regimen. The majority of studies have been single case reports, on a limited number of patients, or have lacked sufficient controls.

Pha rmacotherapy used in the treatment of TTM includes: amitriptyline, ${ }^{8}$ isocarboxide, ${ }^{9}$ clomipramine, ${ }^{4,10}$ lithium, ${ }^{11}$ fluoxetine, ${ }^{4,12,13}$ buspirone, ${ }^{14}$ paroxetine, ${ }^{4}$ pimozide augmentation, ${ }^{15}$ trazodone, ${ }^{16}$ sertraline, ${ }^{17}$ fluvoxamine,${ }^{4,18}$ naltrexo ne,${ }^{4}$ citalo pram,${ }^{4}$ venlafaxine ${ }^{4}$ and inositol. ${ }^{4,19}$

In the treatment of TTM, one study of fluvoxamine found that dosages of less than $300 \mathrm{mg} /$ day had limited efficacy in the treatment of 21 patients over a 12 -week period. ${ }^{18}$

This case study highlights the difficulties experienced by a patient with OCD, TTM and major depression, and indicates the effectiveness of fluvoxamine in the treatment of such a combination.

\section{Case study}

A 19 -year-old law student presented with the complaint of excessive hair pulling leading to extensive hair loss. This behaviour started at the age of 10 years with the pulling of his eyebrows. It subsided at the age of 13 years, only to resurface at the age of 15 years (pulling of scalp hair). This behaviour occurs when he is under pressure from psychosocial problems and even when he is in a subjectively relaxed state. Coinciding with this behaviour the patient complained of an irritable and dysphoric mood, decreased energy and drive, initial insomnia, loss of appetite and weight (approximately $10 \mathrm{~kg}$ in the past 6 months), lack of concentration and forgetfulness, social withdrawal, and suicide ideation (no plans or attempts), over the previous 6 months.

$\mathrm{O} n$ further questioning the patient had obsessions concerning contamination, aggression, hoarding, pathological doubt and a need for symmetry and exactness. His compulsions included cleaning/washing, checking, repeating rituals and ordering/ arranging. For the patient the washing and ordering were the most disturbing, influencing his daily activities and leading to increased anxiety. The Yale Brown 0 bsessive Compulsive Scale (Y-BOCS) Checklist was used to monitor the severity and progress of these obsessions and compulsions.

The diagnosis of TTM and OCD with a co-morbid major depression (moderate to severe) was made. The patient was placed on fluvoxamine (Luvox) at a starting dose of $50 \mathrm{mg}$ nocte and subsequently increased to $100 \mathrm{mg}$ nocte after 1 week and then to 150 $\mathrm{mg}$ nocte the following week. He also received supportive psychotherapy. The patient was followed up at regular intervals and the Hamilton Depression Rating Scale (HAMD) and the Y-BOCS $C$ hecklist were used to monitor his progress. 
The patient had improved scores on the HAM D after 2 weeks on the maintenance dose of $150 \mathrm{mg}$, with the biggest improvement in symptoms after 4 weeks of treatment He continued to improve until remission of the depression was achieved after 8 weeks of treatment The $O C D$ symptoms prevailed for a longer period of time but lessened in severity. After 12 weeks of treatment only mild obsessions and compulsions (washing and ordering) prevailed that were no longer bothersome to the patient. The TTM symptoms increased in severity for the first 4 weeks of treatment and subsided a fter 12 weeks of treatment Full remission of the OCD and TTM could not be commented on, as the patient discontinued his follow -up visits.

\section{Disc ussion}

This case illustrates the early onset of TTM (before the age of 12 years), its waxing and waning course through life with episodes of remission, and the pulling of hair from different sites. The definite increase in severity of hair pulling after the initiation of treatment was a negative finding and the reason for this is currently unknown. This case further highlights the importance of questioning the patient on symptoms of OCD and the exclusion of co-morbid conditions (like depression) when evaluating patients with TTM .

The choice of medication was made largely because of the prominent $O C D$ symptoms and co-morbid depressive symptoms for which fluvoxamine has been proved to be effective. The medication was prescribed as a nocte dosage to help the patient with the insomnia he was experiencing and to prevent daytime sedation that might have influenced his studies and working capacity (therefore the morning dose was omitted). The dosage was taken to a level where the patient experienced clinical benefit, continued improvement in his symptoms and no sideeffects.

The patient did not report any side-effects on the medication. It is therefore unlikely that side-effects contributed to his discontinuation of follow -up visits.
As illustrated, the combination of fluvoxamine and supportive psychotherapy was beneficial in the treatment of this patient's illness. Although it was not possible to determine whether full remission was obtained, sufficient and significant improvement was made.

\section{Conclusion}

Further research into the a etiology of TTM, sufficient treatment protocols for these disorders (TTM as a single entity and in combination with other disorders like OCD and major depressive episode), as well as the long-term treatment options a vailable, are warranted.

\section{References}

1. Kelly LJ. O bsessive compulsive spectrum disorders. Journal of Depression and Anxiety 1999; 2 (1): $54-60$.

2. Jefferys DE. Trichotillomania - it's time to talk. OCD N ews 2001; 9 (1): $2-3$.

3. Stein DJ, Simeon D, C ohen L, Hollander E. Trichotillomania and obsessive-compulsive disorder. J C lin Psychiatry 1995; 56: suppl 4, 28-35.

4. Koran LM . 0 bsessive Compulsive and Related Disorders in Adults. A Comprehensive C linical G uide. C ambridge: C ambridge University Press, 1999: 185-201.

5. Gordon C, Berk M. Trichotillomania: self response from a South African population. Journal of Depression and Anxiety 1999: 2 (1): $36-40$

6. Cohen LJ, Stein DJ, Simeon D, et al. C linical profile, co-morbidity, and treatment history in 123 hair pullers: Asurvey study. J C lin Psychiatry 1995; 56: 319-326.

7. Vogel W, Hull J. Trichotillomania and $O C D: A$ case study highlighting treatment difficulties. Journal of Depression and Anxiety 1999: 2 (4):136-139.

8. Snyder S. Single case study Trichotillomania treated with amitriptyline. J N erv M ent Dis 1980 ; 168: $505-507$.

9. Krishnan RR, Davidson J, Miller R. MAO inhibitor therapy in trichotillomania associated with depression: Case report J C lin Psychiatry 1984; 45: 267-268.

10. Pollard $A C$, Ibe 10, Krojanker DN, Kitchen AD, Bronson SS, Flynn TM. Clomipramine treatment of trichotillomania: a follow-up report on four cases. J C lin Psychiatry 1991; 52: 128 . 130

11. Christenson $G A$, Popkin $M K, M$ ackenzie $T B$, Realmuto $G M$. Lithium treatment of chronic hair pulling. I C lin Psychiatry 1991; 52: 116-120.

12. W inchel RM, Jones IS, Stanley B, M olcho A, Stanley M. C linical characteristics of trichotillomania and its response to fluoxetine. J C lin Psychiatry 1992; 53: 304-308.

13. Streichenwein SM, Thomby Jl. A long term, double-blind, place-controlled crossover trial of the efficacy of fluoxetine for trichotillomania. Am J Psychiatry 1995; 152: 1192-1196.

14. Reid TL Treatment of GAD and trichotillomania with buspirone. Am J Psychiatry 1992; 53: 573.

15. Stein DJ, Hollander E. Low-dose pimozide augmentation of serotonin reuptake blockers in the treatment of trichotillomania. J C lin Psychiatry 1992; 53: 123-126.

16. Sunkureddi K, Markovit P. Trazodone treatment of obsessive compulsive disorder and tichotillomania. Am J Psychiatry 1993; 150: 523

17. Mahmood AR. Trichotillomania associated with HIV infection and response to sertraline. Psychosomatics $1995 ; \mathbf{3 6}: 417-418$

18. Figitt DP, M CClellan KJ. Fluvoxamine: An updated review of its use in the management of adults with anxiety disorders. Drugs 2000; 60: 925-964.

19. SeedatS, Stein DJ, Harvey BH. Inositol in the treatment of trichotillomania and compulsive skin picking. J C lin Psychiatry 2001; 62:60-61. 